\title{
Mosapride Accelerates the Delayed Gastric Emptying of High-Viscosity Liquids: A Crossover Study Using Continuous Real-Time ${ }^{13} \mathrm{C}$ Breath Test (BreathID System)
}

\author{
Yasunari Sakamoto, ${ }^{1}$ Yusuke Sekino, ${ }^{1}$ Eiji Yamada, ${ }^{1}$ Hidenori Ohkubo, ${ }^{1}$ Takuma Higurashi, ${ }^{1}$ Eiji Sakai, ${ }^{1}$ Hiroshi lida, ${ }^{1}$ Kunihiro \\ Hosono, ${ }^{1}$ Hiroki Endo, ${ }^{1}$ Takashi Nonaka, ${ }^{1}$ Tamon Ikeda, ${ }^{1}$ Koji Fujita, ${ }^{1}$ Masato Yoneda, ${ }^{1}$ Tomoko Koide, ${ }^{1}$ Hirokazu Takahashi, \\ Ayumu Goto, ${ }^{1}$ Yasunobu Abe, ${ }^{1}$ Eiji Gotoh, ${ }^{2}$ Shin Maeda, ${ }^{1}$ Atsushi Nakajima ${ }^{1}$ and Masahiko Inamori ${ }^{1,3 *}$ \\ ${ }^{1}$ Gastroenterology Division and ${ }^{2}$ Department of Medical Education, Yokohama City University School of Medicine, Yokohama, Japan; ${ }^{3}$ Office \\ of Postgraduate Medical Education, Yokohama City University Hospital, Yokohama, Japan
}

\section{Background/Aims}

The administration of liquid nutrients to patients is often accompanied by complications such as gastroesophageal reflux. To prevent gastroesophageal reflux, high-viscosity liquid meals are used widely, however, it still remains controversial whether high-viscosity liquid meals have any effect on the rate of gastric emptying. The present study was conducted with the aim of determining whether high-viscosity liquid meals had any effect on the rate of gastric emptying and mosapride might accelerate the rate of gastric emptying of high-viscosity liquid meals.

\section{Methods}

Six healthy male volunteers underwent 3 tests at intervals of $>1$ week. After fasting for $>8$ hours, each subject received one of three test meals (liquid meal only, high-viscosity liquid meal [liquid meal plus pectin] only, or high-viscosity liquid meal 30 minutes after intake of mosapride). $\mathrm{A}^{13} \mathrm{C}$-acetic acid breath test was performed, which monitored the rate of gastric emptying for 4 hours. Using the Oridion Research Software ( $\beta$ version), breath test parameters were calculated. The study parameters were examined for all the 3 test conditions and compared using the Freidman test.

Results

Gastric emptying was significantly delayed following intake of a high-viscosity liquid meal alone as compared with a liquid meal alone; however, intake of mosapride prior to a high-viscosity liquid meal was associated with a significantly accelerated rate of gastric emptying as compared with a high-viscosity liquid meal alone.

\section{Conclusions}

This study showed that high-viscosity liquid meals delayed gastric emptying: however, mosapride recovered the delayed rate of gastric emptying by high-viscosity liquid meals.

(J Neurogastroenterol Motil 2011;17:395-401)

Key Words

Breath test; Gastric emptying; Mosapride; Pectin

Received: July 9, 2011 Revised: August 14, 2011 Accepted: August 17, 2011

(c) This is an Open Access article distributed under the terms of the Creative Commons Attribution Non-Commercial License (http://creativecommons. org/licenses/by-nc/3.0) which permits unrestricted non-commercial use, distribution, and reproduction in any medium, provided the original work is properly cited.

*Correspondence: Masahiko Inamori, MD, PhD

Gastroenterology Division, Yokohama City University School of Medicine, 3-9 Fukuura, Kanazawa-ku, Yokohama 236-0004, Japan

Tel: +81-45-787-2640, Fax: +81-45-784-3546, E-mail: inamorim@med.yokohama-cu.ac.jp

Financial support: None.

Conflicts of interest: None. 


\section{Introduction}

Feeding via a percutaneous endoscopic gastrostomy (PEG) tube is a safe and efficient method for patients who are unable to maintain an adequate oral intake. The administration of liquid nutrients is often accompanied by complications such as vomiting, diarrhea and aspiration pneumonia caused by gastroesophageal reflux (GER), which also presumably causes the vomiting. Over the long-term, aspiration pneumonia has been the most common cause of death in patients fed via a gastrostomy tube. ${ }^{1,2}$ These complications may be minimized if the patient is in the sitting position during the nutrient administration or if the diet is administered at a slower rate. Nevertheless, these methods draw a lot of patience from both the patients and their caregivers, and maintenance of the same body position for many hours may worsen the conditions of patients with pressure ulcers.

The tubing used for PEG feeding enables administration of high-viscosity liquid meals, which would be expected to be associated with a lower likelihood of reflux from the stomach. Rapid administration of high-viscosity nutrients in PEG feeding can reduce the risk of GER substantially, and may eventually contribute to a reduction in the incidence of complications such as aspiration pneumonia with improvements in the quality of life of the patients and their caregivers. ${ }^{3,4}$

In healthy individuals, addition of pectin has been shown to increase the viscosity of enterally administered meals and to accelerate gastric emptying. ${ }^{5}$ On the other hand, gelatinization slowed down the gastric emptying. ${ }^{6}$

Mosapride citrate (mosapride) is a novel gastroprokinetic agent that enhances gastrointestinal motility by stimulating the 5-hydroxytryptamine $4\left(5-\mathrm{HT}_{4}\right)$ receptor. $^{7}$ Stimulation of the $5-\mathrm{HT}_{4}$ receptor causes contractions mediated by cholinergic neurons in isolated ileal preparations. Stimulation of the $5-\mathrm{HT}_{4}$ receptor modulates the motility of the gastrointestinal tract, either increasing it or decreasing it, depending on the animal species and the anatomical region. The $5-\mathrm{HT}_{4}$ receptor-mediated response of acetylcholine release in antral, corporal and fundic strips isolated from guinea pig stomach corresponds to the expression level of the $5-\mathrm{HT}_{4}$ receptor in the myenteric plexus. In vitro receptor autoradiograms of the stomach and colon indicate that the distribution of the $5-\mathrm{HT}_{4}$ receptor in human tissues is similar to that in the guinea pig, even though the density of the $5-\mathrm{HT}_{4}$ receptor in the myenteric plexus in humans is lower than that in the guinea pig myenteric plexus. The $5-\mathrm{HT}_{4}$ receptor in the myenteric plexus may accelerate gastrointestinal motility. Thus, $5-\mathrm{HT}_{4}$ agonists and antagonists may be suitable for the treatment of dysfunction of gastrointestinal motility. ${ }^{8}$

We investigated whether administration of a high-viscosity liquid meal was associated with delayed gastric emptying, and whether administration of mosapride prior to that of a high-viscosity liquid meal accelerated the delayed gastric emptying rate.

\section{Materials and Methods}

\section{Subjects}

The subjects were 6 asymptomatic male volunteers (average age, 29 years; age range: 22-38 years). None of the volunteers were habitual drinkers. All were non-smokers, and none of the subjects had a history of gastrointestinal disease or abdominal surgery. None of the subjects were on medication at the time of the study. None of the subjects were receiving any drugs.

\section{Study Protocol}

Six subjects participated in this randomized, 3-way crossover study. They were randomly assigned to receive a $5 \mathrm{mg}$ mosapride tablet 30 minutes before the high-viscosity liquid meal or a highviscosity liquid meal alone without prior administration of mosapride, or a test meal alone. Each of the test conditions were separated by a washout period of at least 7 days. In each test condition, the breath test was performed after overnight fasting (for at least 8 hours); the monitoring was performed for 4 hours while the subjects remained in the sitting position.

\section{Test Meals}

The test meal (control) was a $400 \mathrm{kcal} / 400 \mathrm{~mL}$ liquid meal (Racol with milk flavor, Otsuka Pharmaceutical Co, Ltd, Tokyo, Japan) containing $100 \mathrm{mg}$ of ${ }^{13} \mathrm{C}$-acetic acid (Cambridge Isotope laboratories, Inc, Boston, USA). For a high-viscosity liquid meal (thick liquid), pectin (16 g of Toromi Perfect; Nissin Oilio Co, Ltd, Tokyo, Japan), which was used to increase the viscosity, was added to the test meal. The meal had the following composition in a volume of $16 \mathrm{~g}$ : protein $0.10 \mathrm{~g}$, glucose $9.0 \mathrm{~g}$, fiber $5.3 \mathrm{~g}$, Na $224 \mathrm{mg}$ and $\mathrm{K} 101 \mathrm{mg}$. The total energy content was only $37 \mathrm{kcal}$. When pectin was mixed into the liquid meal, it was gelatinized and its viscosity increased. The viscosity of the liquid meal for enteral nutrition was 6 milipascal seconds $(\mathrm{mPa} \cdot \mathrm{s})$ versus 2000 $\mathrm{mPa} \cdot \mathrm{s}$ for the high-viscosity liquid meal, while the calorie contents of the 2 meals were 400 and $428 \mathrm{kcal}$, respectively. ${ }^{5}$ The test 
meals (control and thick liquid) were consumed within $5 \mathrm{mi}-$ nutes.

\section{${ }^{13}$ C-Acetic Acid Breath Test}

Breath samples were continuously collected via a nasal tube using the BreathID system (Oridion Medical Ltd, Jerusalem, Israel), first at the baseline before the test meal administration, and then following completion of the test meal administration (time 0 ) and for 4 hours thereafter.

\section{Data Analysis}

The data were analyzed using the Oridion Research Software, $\beta$ version (Oridion Medical Ltd). The time versus ${ }^{13} \mathrm{CO}_{2}$ excretion rate curve was fitted to the conventional formula of $y(t)$ $=\mathrm{m}\left(1-\mathrm{e}^{-\mathrm{kt}}\right)^{\beta-1}$, and the regression-estimated constants of $\kappa$ and $\beta$ were determined. After the mathematical analysis, the time required for emptying of $50 \%$ of the labeled meals $\left(T_{1 / 2}\right)$, the ana$\log$ to the scintigraphy lag time for $10 \%$ emptying of the labeled meal $\left(\mathrm{T}_{\text {lag }}\right)$, the gastric emptying coefficient (GEC) and the regression-estimated constants ( $\kappa$ and $\beta$ ) were calculated. ${ }^{9-17}$

\section{Statistical Methods}

Statistical evaluation was performed using the Friedman test and the Wilcoxon signed rank test. The level of significance was set at $P<0.05$. Statistical analyses were performed using the StatView software (SAS Institute, Cary, NC, USA).

\section{Ethics}

The study was conducted in accordance with the Declaration of Helsinki. The study protocol using the BreathID system was approved by the Ethics Committee of Yokohama City University School of Medicine.

\section{Results}

Six male subjects (mean age, 29 years old; median age, 28.5 years old; range, 22-38 years) completed this study. The subject's heights and weights were as follows: mean height, $169.3 \mathrm{~cm}$; median height, $169 \mathrm{~cm}$; height range, $165-173 \mathrm{~cm}$; mean weight, 75 $\mathrm{kg}$; median weight, $71 \mathrm{~kg}$; and weight range, 59-104 kg. No adverse events occurred during the study.

Table and Figures summarize the high-viscosity and mosapride-induced changes in the breath test parameters. When the gastric emptying parameters were compared among the 3 testmeal conditions, the $T_{1 / 2}, T_{\text {lag }}$, GEC and $\kappa$ value were significantly different. In view of GEC, gastric emptying was sig-

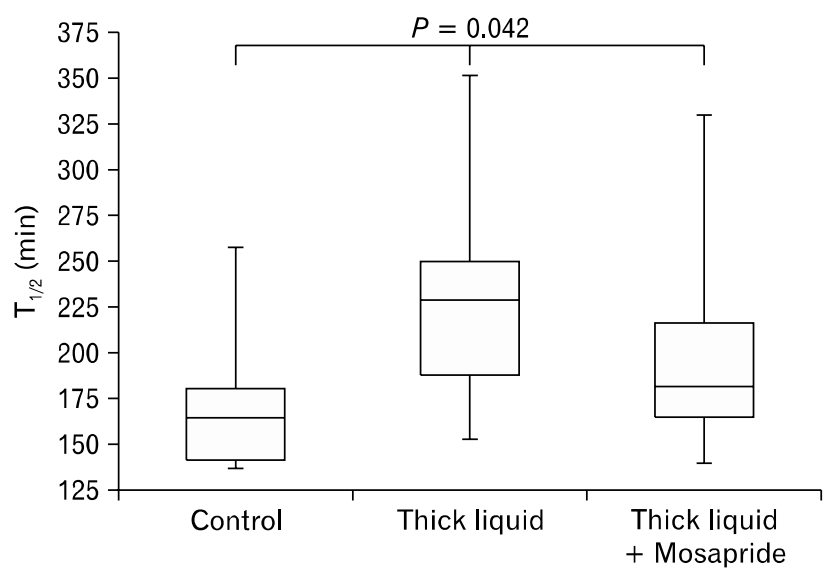

Figure 1. The time required for emptying of $50 \%$ of the labeled meal $\left(\mathrm{T}_{1 / 2}\right)$ was higher after administration of the high-viscosity liquid meal alone than after administration of the high-viscosity liquid meal plus mosapride ( $P=0.042$, Friedman's test; $P=0.075$, Wilcoxon signed rank test [Control vs Thick liquid]; $P=0.345$, Wilcoxon signed rank test [Control vs Thick liquid + Mosapride]; $P=0.028$, Wilcoxon signed rank test [Thick liquid vs Thick liquid + Mosapride]).

Table. Comparison of Breath Test Parameters

\begin{tabular}{lcccc}
\hline Parameter & Control & Thick liquid & Thick liquid plus mosapride & $P$-value \\
\hline $\mathrm{T}_{1 / 2}$ & $163.7(135.6-266.3)$ & $227.9(149.0-363.3)$ & $180.4(136.3-342.1)$ & 0.042 \\
$\mathrm{~T}_{\text {lag }}$ & $103.3(76.5-124.4)$ & $119.3(80.5-206.1)$ & $100.6(73.7-205.9)$ & 0.030 \\
$\mathrm{GEC}$ & $3.09(2.64-3.57)$ & $2.42(1.37-3.00)$ & $2.90(1.26-3.46)$ & 0.030 \\
$\beta$ & $2.16(1.65-3.65)$ & $1.84(1.66-2.47)$ & $2.00(1.78-2.20)$ & 0.223 \\
$\kappa$ & $0.48(0.24-0.78)$ & $0.29(0.20-0.48)$ & $0.41(0.23-0.52)$ & 0.042 \\
\hline
\end{tabular}

$\mathrm{T}_{1 / 2}$, time for emptying of $50 \%$ of the labeled meals (min); $\mathrm{T}_{\text {lag }}$, analog to the scintigraphy lag time for $10 \%$ emptying of the labeled meal (min); GEC, gastric emptying coefficient; $\beta$ and $\kappa$, regression-estimated constants.

A larger (smaller) value of $\beta$ indicates slower (faster) emptying during the early phase, and a larger (smaller) value of $\kappa$ indicates faster (slower) emptying during the later phase. All values are median (range). Statistical evaluation was performed using the Friedman test. 


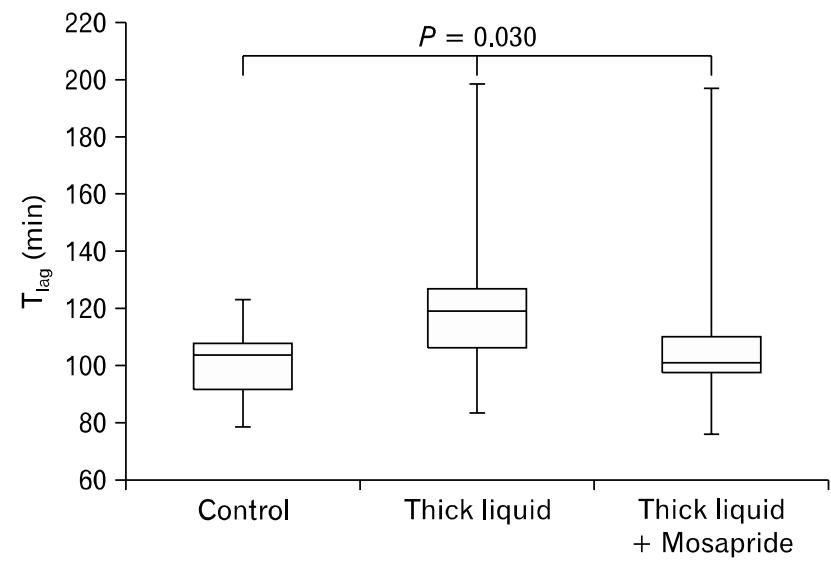

Figure 2. The analog to the scintigraphy lag time for $10 \%$ emptying of the labeled meal $\left(\mathrm{T}_{\mathrm{lag}}\right)$ was higher after administration of the high-viscosity liquid meal alone than after administration of the high-viscosity liquid meal plus mosapride ( $P=0.030$, Friedman's test; $P=0.116$, Wilcoxon signed rank test [Control vs Thick liquid]; $P=$ 0.463 , Wilcoxon signed rank test [Control vs Thick liquid + Mosapride]; $P=0.028$, Wilcoxon signed rank test [Thick liquid vs Thick liquid + Mosapride]).

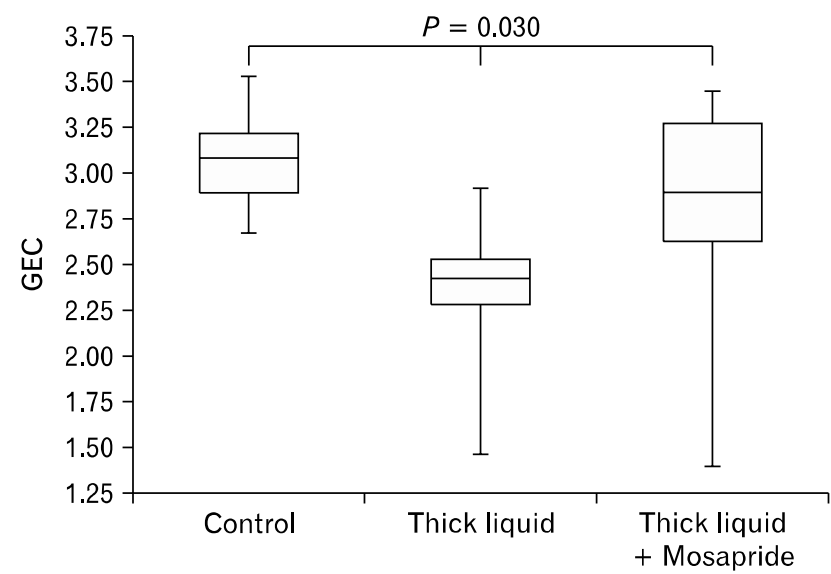

Figure 3. The gastric emptying coefficient (GEC) was lower after administration of the high-viscosity liquid meal alone than after administration of either the control meal $(P=0.030$, Friedman's test; $P$ $=0.028$, Wilcoxon signed rank test [Control vs Thick liquid]; $P=$ 0.463 , Wilcoxon signed rank test [Control vs Thick liquid + Mosapride]; $P=0.075$, Wilcoxon signed rank test [Thick liquid vs Thick liquid + Mosapride]).

nificantly delayed in the high-viscosity meal alone group compared with the control meal group (Fig. 3). Gastric emptying with the high-viscosity liquid meal was accelerated statically by mosapride in $\mathrm{T}_{1 / 2}, \mathrm{~T}_{\text {lag }}$ and $\kappa$ (Fig. 1-3 and 5).

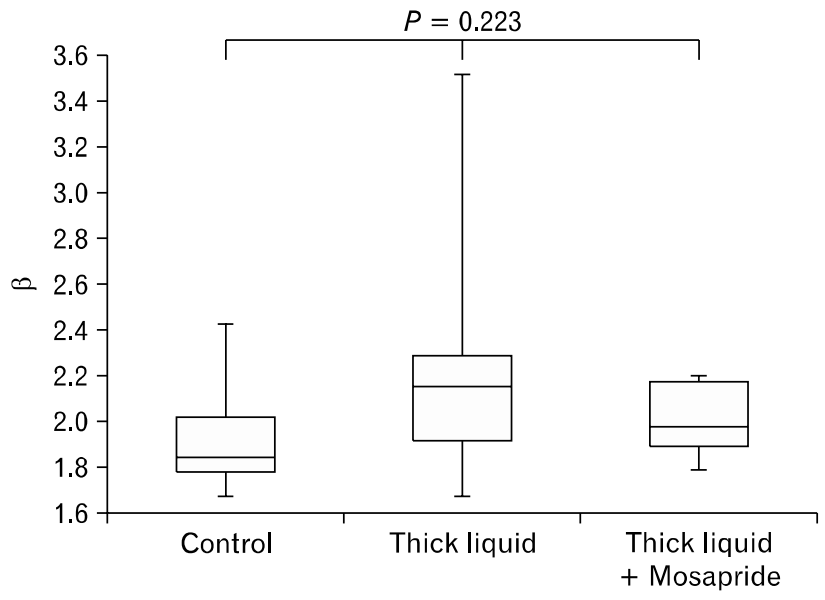

Figure 4. No significant differences in the value of the regressionestimated constants $(\beta)$ were observed among the 3 conditions (highviscosity liquid meal alone, control meal, and high-viscosity liquid meal plus mosapride $(P=0.223$, Friedman test; $P=0.046$, Wilcoxon signed rank test [Control vs Thick liquid]; $P=0.645$, Wilcoxon signed rank test [Control vs Thick liquid + Mosapride]; $P=0.463$, Wilcoxon signed rank test [Thick liquid vs Thick liquid + Mosapride]).

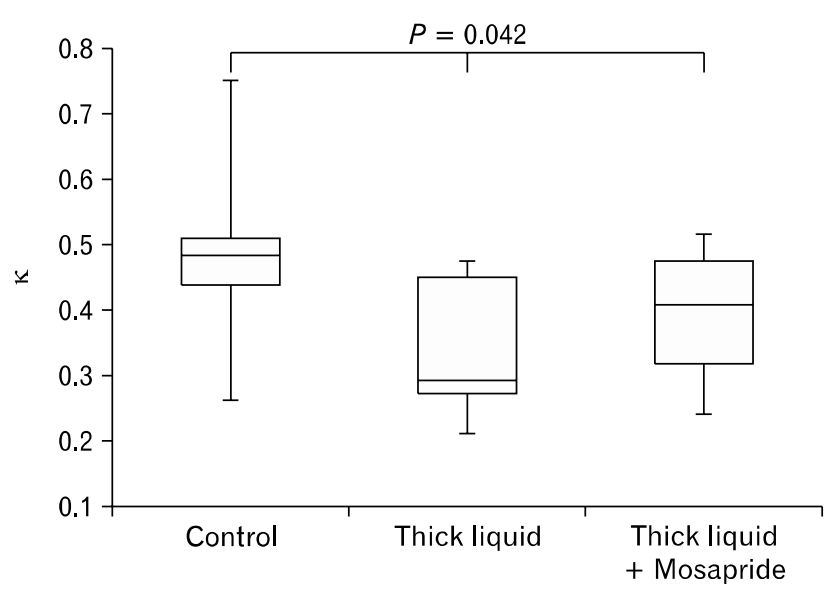

Figure 5. A lower value of the regression-estimated constants ( $\kappa)$ was obtained after administration of a high-viscosity liquid meal alone than after administration of the high-viscosity liquid meal plus mosapride $(P$ $=0.042$, Friedman's test; $P=0.075$, Wilcoxon signed rank test [Control vs Thick liquid]; $P=0.249$, Wilcoxon signed rank test [Control vs Thick liquid + Mosapride $] ; P=0.028$, Wilcoxon signed rank test [Thick liquid vs Thick liquid + Mosapride]).

\section{Discussion}

Food particles must be broken down to a certain size before they can be emptied from the stomach; study conducted in dogs showed that food was broken down to particles smaller than 7 
$\mathrm{mm}$ in size before it was emptied from the stomach. Gelatinization transiently increases the size of liquid meal particles; thus it is known that more time is required for gelatinized meals to be broken down, which results in prolonged gastric emptying. ${ }^{6}$

The present study was conducted to examine the changes in the gastric emptying rate induced by mosapride during the first 4 hours after ingestion of a control liquid meal or gelatinized liquid meal (high-viscosity liquid meal) in healthy subjects. The study results revealed that gelatinization slowed down the gastric emptying, while mosapride accelerated the gastric emptying. ${ }^{6}$

On the other hand, Shimoyama et $\mathrm{al}^{5}$ reported that gelatinized liquid meals, which contained pectin, increased the viscosity of enteral nutrients and accelerated gastric emptying. The conflicting results may be explained by the differences in volume and fat content of test meals and in the viscosity of test meals employed. ${ }^{5}$

Dietary fibers such as psyllium and guar gum have been shown to delay the rate of gastric emptying of both liquids and solids, presumably via increasing the viscosity of meals. Russell and Bass ${ }^{18,19}$ performed a study to determine whether the changes in the rate of gastric emptying of viscous psyllium and guar gum meals were associated with antroduodenal motility changes. Dogs were surgically fitted with mid-duodenal cannulas for the measurement of gastric emptying rate. Strain-gauge force transducers were used to monitor the antral and duodenal contractile responses to the test meals. More rapid gastric emptying of a low-viscosity fiber meal from the stomach as compared with that of a high-viscosity meal was noted. However, despite the difference in the gastric emptying time, neither of the test meals stimulated antral or duodenal motility. They concluded that the gastric emptying rate decreased as the fiber content and viscosity of the test meals increased, and that the viscosity-related delay in gastric emptying was not due to any effect on the postprandial antroduodenal motility. ${ }^{18,19}$

$\mathrm{Xu}$ et $\mathrm{al}^{20}$ examined the effect of enhanced viscosity on the gastric emptying rate in dogs by simultaneously monitoring the gastric and intestinal myoelectrical recordings for 2 hours. They concluded that increase in the viscosity of a liquid meal by galactomannan was associated with a significant decrease in the rate of gastric emptying in the dog, along with an increase in the frequency and strength of intestinal contractions, but had no effect on the intestinal slow wave rhythms. ${ }^{20}$

Holt et $\mathrm{al}^{21}$ studied the rate of paracetamol absorption in relation to the rate of gastric emptying in healthy volunteers. The same parameters were examined after the addition of guar gum and pectin, both of which increased the viscosity, to the ingested paracetamol. Both the rate of gastric emptying and the rate of paracetamol absorption decreased in association with the increase in the viscosity of ingested drug solution, however, no significant decrease in the total absorption of drug, as reflected by the urinary recovery, was observed. The results suggest that the effects of guar gum and pectin on paracetamol absorption could be attributable simply to the alteration in the rate of gastric emptying. ${ }^{21}$

Sandhu et $\mathrm{al}^{22}$ studied the rate of gastric emptying in healthy volunteers. They examined the gastric emptying rates of a liquid and a solid meal (with or without $15 \mathrm{~g}$ of pectin) by a radioisotope technique using ${ }^{99 \mathrm{~m}} \mathrm{Tc}$-dithiopropylthiomine or a perfused catheter system. From their results, they concluded that pectin supplementation delayed the rate of gastric emptying of both the liquid and solid meals in the normal human subjects without causing any notable changes in the gastroduodenal motility or significant variations in the plasma levels of the pancreatic hormones. They suggested that the effect of pectin on the rate of gastric emptying may be caused simply by the increase in the viscosity of meals. ${ }^{22}$

Thus, a number of studies concluded that the gastric emptying rate decreases as the viscosity of test meals increases. This is likely attributable to the increase of friction between the gastric mucosa and the test meal associated with increased viscosity. Shimoyama et $\mathrm{al}^{5}$ reported accelerated gastric emptying of a high-viscosity liquid meal, however, the viscosity of their test meal was 900 centipoises (cP), which is equal to $900 \mathrm{mPa} \cdot \mathrm{s}$. In our study, we used a pectin-added liquid meal with viscosity of 2,000 $\mathrm{mPa} \cdot \mathrm{s}$. Thus, the lower viscosity may explain accelerated gastric emptying in Shimoyama et al's study. ${ }^{5}$ Also, we used $16 \mathrm{~g}$ of pectin, which was similar to the volume of pectin used in the study by Sandhu et $\mathrm{al}^{22}$ (15 g of pectin), and our results were similar to theirs.

We cannot ignore the effect of mosapride, one of the pharmacological actions of which is acceleration of gastric emptying.

The evaluation of gastric emptying using the ${ }^{13} \mathrm{C}$-acetic acid breath test is a noninvasive method. We measured gastric emptying using the BreathID system, which collects continuous breaths. The subject ingests ${ }^{13} \mathrm{C}$-labeled acetic acid, which passes through the stomach and is absorbed in the duodenum and superior small bowel. The ${ }^{13} \mathrm{C}$-labeled acetic acid is then metabolized in the liver and excreted from the lungs as ${ }^{13} \mathrm{CO}_{2}$. This pathway enables gastric emptying to be measured in a noninvasive manner. ${ }^{13}$ Value of breath test parameters is influenced by method (for example, radioisotope method or breath test), test meal 
(solid or liquid), label (acetate or octanete) and subjects (healthy volunteers or patients). Our studies using breathID with $400 \mathrm{kcal}$ per $400 \mathrm{~mL}$ liquid meal containing $100 \mathrm{mg}$ of ${ }^{13} \mathrm{C}$-acetic acid was original and we had little data, ${ }^{12}$ but $200 \mathrm{kcal}$ per $200 \mathrm{~mL}$ liquid meal containing $100 \mathrm{mg}$ of ${ }^{13} \mathrm{C}$-acetic acid usually showed $\mathrm{T} 1 / 2$ of about 100 minutes. ${ }^{10,11,14-17}$

Shimoyama et $\mathrm{al}^{5}$ reported that high-viscosity liquid meal of $900 \mathrm{mPa}$.s accelerated gastric emptying, whereas our test meal of 2,000 mPa.s, delayed gastric emptying. Many factors influence gastric emptying: the blood glucose, ${ }^{23}$ insulin, ${ }^{24}$ gastrin, ${ }^{25,26}$ meal fat, ${ }^{27}$ cholecystokinin ${ }^{28}$ and the viscosity of meal. But without mosapride, Shimoyama's viscosity may be suitable for PEG feeding to accelerate gastric emptying and to prevent GER.

In conclusion, this study showed that high-viscosity liquid meals delayed gastric emptying in healthy volunteers: however, mosapride recovered the delayed rate of gastric emptying by high-viscosity liquid meals. The clinical implications of our results remain unclear; however, our findings suggest that high-viscosity liquid meals with mosapride may be useful in some clinical settings for patients with feeding via gastrostomy.

\section{Acknowledgements and Disclosures}

None of the authors have any disclosures to make.

Hiroshi Iida, Hiroki Endo, Yusuke Sekino, Kunihiro Hosono, Yasunari Sakamoto, Tomoko Koide, Hirokazu Takahashi, Chikako Tokoro, Ayumu Goto, Yasunobu Abe, Shin Maeda, Atsushi Nakajima, Masahiko Inamori.

Hiroshi Iida, Yusuke Sekino, Yasunari Sakamoto and Tomoko Koide performed the research, Hirokazu Takahashi, Chikako Tokoro, Ayumu Goto and Yasunobu Abe analyzed the data and wrote the paper, Shin Maeda amd Atsushi Nakajima contributed the capsule endoscopy for the study and Masahiko Inamori designed the research study and wrote the paper.

\section{References}

1. Coben RM, Weintraub A, DiMarino AJ Jr, Cohen S. Gastroesophageal reflux during gastrostomy feeding. Gastroenterology 1994; 106;13-18.

2. Marik PE. Aspiration pneumonitis and aspiration pneumonia. $\mathrm{N}$ Engl J Med 2001;344:665-671.

3. Kanie J, Suzuki Y, Akatsu H, Kuzuya M. Prevention of late complications by half-solid enteral nutritents in percutaneous endoscopic gastrostomy tube feeding. Gerontology 2004;50:417-419.

4. Kanie J, Suzuki Y, Iguchi A, Akatsu H, Yamamoto T, Shimokata H. Prevention of gastroesophageal reflux using an application of half- solid nutrients in patients with percutaneous endoscopic gastrostomy feeding. J Am Geriatr Soc 2004;52:466-467.

5. Shimoyama Y, Kusano M, Kawamura O, et al. High-viscosity liquid meal accerates gastric emptying. Neurogastroenterol Motil 2007;19: 879-886.

6. Kawasaki N, Suzuki Y, Urashima M, et al. Effect of gelatinization on the esophagastric emptying and obsorption. Hepatogastroenterology 2008;55:1843-1845.

7. Yoshida N. [Pharmacological effects of the gastriprokinetic agent mosapride citrate.] Nihon Yakurigaku Zasshi 1999;113:299-307. [Japanese]

8. Taniyama K, Makimoto N, Furuichi A, et al. Functions of peripheral 5-hydroxytryptamine receptors, especially 5-hydroxytryptamine4 receptor, in gastrointestinal motility. J Gastroenterol 2000;35:575582.

9. Ghoos YF, Maes BD, geypens BJ, et al. Measurement of gastric emptying rate of solids by means of a carbon-labeled octanoic acid breath test. Gastroenterology 1993;104:1640-1647.

10. Inamori M, Akiyama T, Akimoto K, et al. Early effects of peppermint oil on gastric emptying: a crossover study using a continuous real-time ${ }^{13} \mathrm{C}$ breath test (BreathID system). J Gastroenterol 2007;42: 539-542.

11. Inamori $\mathrm{M}$, Iida $\mathrm{H}$, endo $\mathrm{H}$, et al. Aperitif effects on gastric emptying: a crossover study using a continuous real-time ${ }^{13} \mathrm{C}$ breath test (BreathID system). Dig Dis Sci 2009;54:816-818.

12. Sakamoto Y, Kato S, Sekino Y, et al. Effects of domperidone on gastric emptying: a crossover study using a continuous real-time ${ }^{13} \mathrm{C}$ breath test (BreathID system). Hepatogastroenterology 2011;58: 637-641.

13. Ikeda $\mathrm{T}$, Inamori $\mathrm{M}$, Fujisawa $\mathrm{N}$, et al. Effects of body positions on gastric emptying with enteral nutrition: a crossover study using a continuous real time ${ }^{13} \mathrm{C}$ breath test (BreathID system). Hepatogastroenterology 2008;5 5:1905-1907.

14. Akimoto $\mathrm{K}$, Inamori $\mathrm{M}$, Iida $\mathrm{H}$, et al. Does postprandial coffee intake enhance gastric emptying?: a crossover study using continuous real time ${ }^{13} \mathrm{C}$ breath test (BreathID system). Hepatogastroenterology 2009;56:918-920.

15. Nonaka T, Kessoku T, Ogawa Y, et al. Does postprandial itopride intake affect gastric emptying?: A crossover study using the continuous real time ${ }^{13} \mathrm{C}$ breath test (BreathID system). Hepatogastroenterology 2011;58:224-228.

16. Sakamoto $Y$, Kato S, Sekino Y, et al. Change of gastric emptying with chewing gum: evaluation using a continuous real-time ${ }^{13} \mathrm{C}$ breath test (BreathID system). J Neurogastroenterol Motil 2011;17:174-179.

17. Nonaka T, Kessoku T, Ogawa Y, et al. Effects of histamine-2 receptor antagonists and proton pump inhibitors on the rate of gastric emptying: a crossover study using a continuous real-time ${ }^{13} \mathrm{C}$ breath test (BreathID system). J Neurogastroenterol Motil 2011;17:287293.

18. Russell J, Bass P. Canine gastric emptying of fiber meals: influence of meals viscosity and antruduodenal motility. Am J Physiol 1985; 249(6 Pt 1):G662-G667.

19. Russell J, Bass P. Method for the quantitation of gastric emptying time of gel test meals. Am J Clin Nutr 1984;40:647-653.

20. Xu X, Brining D, Rafiq A, Hayes J, Chen JD. Effects of enhanced viscosity on canine gastric and intestinal motility. J Gastroenterol 
Hepatol 2005;20:387-394.

21. Holt S, Heading RC, Carter DC, Prescott LF, Tothill P. Effect of gel fibre on gastric emptying and absorption of glucose and paracetamol. Lancet 1979;1:636-639.

22. Sandhu KS, el Samahi MM, Mena I, Dooley CP, Valenzuela JE. Effect of pectin on gastric emptying and gastroduodenal motility in normal subjects. Gastroenterology 1987;92:486-492.

23. Blackburn NA, Redfern JS, Jarjis H, et al. The mechanism of action of guar gum in improving glucose tolerance in man. Clin Sci (Lond) 1984;66:329-336.

24. Torsdottir I, Alpsten M, Holm G, Sandberg AS, Tölli J. A small dose of soluble alginate-fiber affects postprandial glycemia and gas- tric emptying in humans with diabetes. J Nutr 1991;121:795-799.

25. Dozois RR, Kelly KA. Effect of a gastrin pentapeptide on canine gastric emptying of liquids. Am J Physiol 1971;221:113-117.

26. Misiewicz JJ, Holdstock DJ, Waller SL. Motor response of the human alimentary tract to near maximal infusion of pentagastrin. Gut 1967;8:463-469.

27. Stacher G, Bergmann H, Gaupmann G, et al. Fat preload delays gastric emptying: reversal by cisapride. Br J Clin Pharmacol 1990;30: 839-845.

28. Kusano M, Minashi K, Maeda M, et al. Postprandial water intake inhibits gastric antral motility with increase of cholecystokinin in humans. Scand J Gastroenterol 2005;40:1176-1181. 